\title{
Comportement métabolique de Lactococcus lactis en réponse à l'environnement
}

\author{
Christel Garrigues, Myriam Mercade, Pascal Loubière, \\ Nic D. Lindley, Muriel Cocaign-Bousquet*
}

\begin{abstract}
Centre de bio-ingénierie Gilbert-Durand, UMR CNRS/Insa 5504, laboratoire associé Inra, Institut national des sciences appliquées, complexe scientifique de Rangueil, 31077 Toulouse cedex 4, France
\end{abstract}

\begin{abstract}
Metabolic behaviour of Lactococcus lactis in response to environmental conditions. Growth and metabolism of the lactic acid bacteria are closely dependent on the nutritional environment. Major environmental constraints, when they are not directly related to the bacterial growth, are the nature and the availability of the nitrogen and carbon sources. In this paper, the influence of the carbon substrate (sugar) on the metabolism of Lactococcus lactis NCDO 2118 was studied from a kinetic and enzymatic point of view. An original model of metabolism control has been developed and was successively tested in other nutritional environments. This model differs from that generally assumed for lactic acid bacteria and can be applied to other lactococci. Fructose-1,6-bisphosphate plays only a minor role in controlling the further metabolism of pyruvate and NADH/NAD ratio exerts the major control over glycolytic flux. The metabolic consequences of co-substrate utilization were analyzed and the role of serine utilization on the metabolism of L. lactis NCDO 2118 was investigated in more detail. (c) Inra/Elsevier, Paris.
\end{abstract}

\section{Lactococcus lactis / growth / homolactic and mixed metabolism / co-substrate}

Résumé - La croissance ainsi que le métabolisme des bactéries lactiques sont fortement dépendants de l'environnement nutritionnel. Les contraintes environnementales majeures, lorsqu'elles ne sont pas liées à la croissance même du micro-organisme, concernent la nature et la disponibilité de la source d'azote et de carbone. Plus particulièrement, l'effet de la source de carbone (sucre) sur le métabolisme de Lactococcus lactis NCDO 2118 sera analysé d'un point de vue cinétique et enzymatique. Un modèle de régulation original, très différent de celui généralement admis chez les bactéries lactiques, sera proposé. II n'accorde qu'un rôle mineur au fructose-1,6-diphosphate et est basé sur un contrôle strict de la glycolyse par le ratio NADH/NAD. Ce modèle a ensuite été validé dans des conditions de culture différentes et a pu être facilement généralisé à d'autres souches de lactocoques. Puis l'effet des cosubstrats sur le métabolisme des bactéries lactiques sera abordé et le rôle de la sérine dans le métabolisme de $L$. lactis NCDO 2118 sera développé plus précisément. (C) Inra/Elsevier, Paris.

\section{Lactococcus lactis / croissance / métabolisme homolactique et mixte / cosubstrat}

\footnotetext{
* Correspondance et tirés à part
} 


\section{INTRODUCTION}

Les bactéries lactiques, comme tous les micro-organismes, possèdent un métabolisme soumis à des régulations imposées par les contraintes environnementales. Il convient de distinguer deux types de stress différents ayant un rôle majeur dans le comportement de ces bactéries. Le premier se caractérise par des contraintes relevant directement de l'activité microbienne. En effet, la croissance des bactéries lactiques est directement couplée à l'accumulation de produits de fermentations inhibiteurs (acides organiques, bactériocines) et, dans le cas de procédés industriels, à l'acidification du milieu de culture. Ces contraintes se développent et s'accentuent donc tout le long de la fermentation, en ayant des répercussions diverses sur le métabolisme. Il est parfois difficile d'attribuer une cause unique à ce type de stress. L'accumulation d'acide lactique, par exemple, provoque une inhibition de la croissance sans que la cause soit encore bien caractérisée. Des effets sur l'énergétique des cellules dus ầ l'effet protonophore sont incontestables, cependant l'acide lactique provoque aussi une augmentation de la pression osmotique ainsi que l'acidification du milieu. Récemment, Loubière et al. [7] ont montré que l'effet inhibiteur de l'acide lactique rajouté sous forme de sel de lactate dans les cultures de Lactococcus lactis n'était pas significativement différent de celui des simples sels, confirmant ainsi le rôle majeur de la pression osmotique sur la croissance des bactéries lactiques. Concernant l'acidification du milieu, des données apparemment contradictoires sont recensées dans la littérature. La diminution du $\mathrm{pH}$ dans la culture n'est pas inhibitrice en tant que telle, si les cellules sont capables de maintenir un $\mathrm{pH}$ intracellulaire proche de la neutralité. Cependant, une telle homéostasie nécessite une dépense énergétique importante qui impose un régime métabolique où l'énergie devient limitante pour la croissance. Ce mécanisme est fondamentalement différent des effets d'inhibition qui sont liés à l'altération de l'activité des enzymes aux valeurs de $\mathrm{pH}$ éloignées de la neutralité. Le fonctionnement des enzymes du métabolisme, qui conditionne le comportement global de la bactérie, dépend donc de la capacité du micro-organisme à maintenir son $\mathrm{pH}$ intracellulaire, exception faite des enzymes exocellulaires (peptidases, etc.) qui sont directement soumises à l'influence du $\mathrm{pH}$ du milieu. Des travaux dans ce domaine ont montré que la nature de l'acidité a une influence considérable sur le type de réponse obtenu. Par exemple, l'accumulation d'acide lactique provoque une dissipation de gradient transmembranaire plus importante que les acides inorganiques pour un même $\mathrm{pH}$ [1]. L'étude de la réponse d'une bactérie à un stress physicochimique donné relève donc d'une grande complexité et nécessite de bien caractériser la nature de la réponse métabolique, d'autant plus qu' une cascade d'événements peut même se produire pour un seul et même stress. Par exemple, l'accumulation d'acide lactique doit dans un premier temps provoquer une augmentation des besoins en ATP et donc une chute du taux de croissance, sans forcément occasionner une diminution de la vitesse de consommation de sucre. Lorsque le gradient de $\mathrm{pH}$ transmembranaire ne peut plus être maintenu, un deuxième phénomène risque d'être déclenché avec des conséquences très différentes. La chute du $\mathrm{pH}$ intracellulaire modifie considérablement l'environnement des enzymes et leur capacité à maintenir une activité adéquate. Dans ces conditions, le réseau métabolique pourra être considérablement modifié ainsi que sa structure de contrôle : certaines voies métaboliques vont rester actives pendant que d'autres vont cesser de fonctionner. Il est probable que ce type de régulation soit impliqué dans la post acidification de certains produits laitiers. 
Le deuxième type de contraintes environnementales ayant un rôle important dans le développement des bactéries lactiques concerne la disponibilité dans le milieu de culture des nutriments nécessaires à la croissance. En dehors de la présence des sels inorganiques et des vitamines, les contraintes majeures sont imposées par les composés organiques azotés (peptides, acides aminés, bases...) et substrats carbonés. Seul ce dernier point sera traité en détail dans cet article et l'effet de la nature de la source de carbone sur le métabolisme de L. lactis NCDO 2118 sera développé. La source de carbone fournit l'énergie nécessaire aux réactions anaboliques (fonction catabolique), mais constitue aussi une source de carbone pour ces mêmes voies anaboliques, particulièrement si la composition du milieu de culture est réduite [9].

\section{RÔLE DES SUCRES}

L. lactis, et plus généralement les bactéries lactiques homolactiques, sont couramment rencontrées dans l'industrie agro-

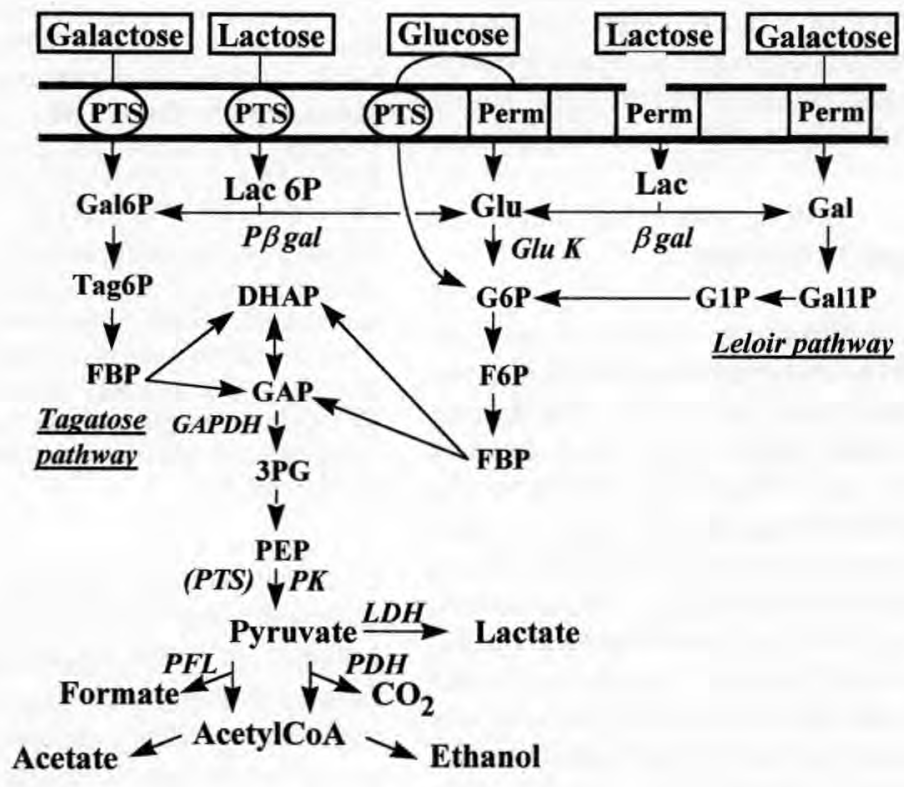

Figure 1. Voies métaboliques impliquées dans l'entrée et le métabolisme du glucose, du galactose et du lactose chez Lactococcus lactis. PTS, sucre phosphotransférase ; Perm, perméases ; Gal6P, galactose-6-phosphate ; Lac6P, Lactose-6-phosphate ; Tag6P, tagatose-6-phosphate ; TDP, tagatose-1,6-diphosphate ; FDP, fructose-1,6-diphosphate ; P $\beta$ gal, phospho- $\beta$-galactosidase ; $\beta$ gal, $\beta$-galactosidase ; GAPDH, glycéraldehyde 3-phophate déshydrogénase ; PK, pyruvate kinase ; LDH, lactate déshydrogénase ; PDH, pyruvate déshydrogénase ; PFL, pyruvate formiate lyase.

Figure 1. Metabolic pathways involved in the uptake and metabolism of glucose, galactose, and lactose by $L$. lactis. Perm, permease-mediated sugar uptake; Gal6P, galactose-6-phosphate; Lac6P, lactose-6-phosphate; TDP, tagatose-1,6-diphosphate; FDP, fructose-1,6-diphosphate; P $\beta$ gal, phospho- $\beta$-galactosidase; $\beta$ gal, $\beta$ galactosidase; GAPDH, glyceraldehyde 3-phophate dehydrogenase; $\mathrm{PK}$, pyruvate kinase; $\mathrm{LDH}$, lactate dehydrogenase; $\mathrm{PDH}$, pyruvate dehydrogenase; PFL, pyruvate formate lyase. 
alimentaire. Elles sont notamment utilisées dans l'industrie laitière pour leur capacité à produire de l'acide lactique (rendement $>90 \%$ ) en excès de sucre. Dans certaines conditions cependant, ces bactéries homolactiques peuvent présenter un métabolisme diversifié, appelé métabolisme mixte, au cours duquel des quantités importantes de formiate, d'acétate et d'éthanol sont produites (figure l). Les produits du métabolisme mixte sont pourtant plus inhibiteurs que le lactate [7]. Le métabolisme mixte a d'abord été observé par Thomas [12] lors de cultures de L. lactis en mode continu, limitées par le glucose. Cependant, malgré l'importance de ce métabolisme, peu d'études sont disponibles dans la littérature et de nombreuses incertitudes persistent encore sur la nature des régulations métaboliques impliquées.

\subsection{Aspect cinétique}

En condition d'excès de substrat, les bactéries homolactiques peuvent présenter un métabolisme mixte mais uniquement sur certains sucres. En effet, avec la souche L. lactis NCDO 2118 cultivée en culture discontinue sur un milieu synthétique non limitant, le milieu MS10 [2], un métabolisme homolactique a été démontré pour les substrats permettant une croissance rapide, comme le glucose, tandis qu'un métabolisme mixte est observé pour les substrats offrant de plus faibles caractéristiques de croissance (figure 2). Des quantités équivalentes de lactate et de formiate plus acétate et éthanol sont produites lors de la croissance sur galactose. Par contre, le métabolisme sur lactose est à souligner pour sa production de lactate extrêmement faible et celle de formiate, d'acétate et d'éthanol proche du maximum théorique [3]. Le degré de diversité du métabolisme semble être lié chez L. lactis NCDO 2118 à la valeur du flux glycolytique, c'est-à-dire à la vitesse de consommation du sucre (tableau I). En

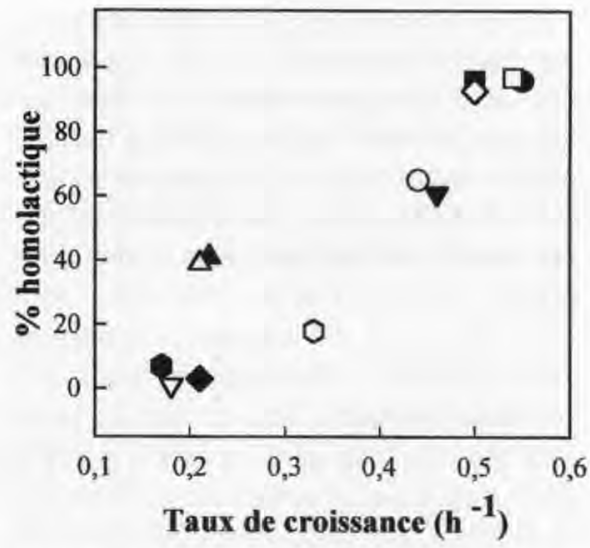

Figure 2. Relation entre le taux de croissance et la quantité de pyruvate transformé en lactate lors de cultures discontinues de L. lactis NCDO 2118 sur divers substrats. Fructose $(\bullet)$;

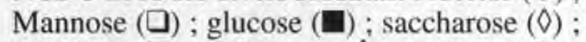
tréhalose $(\boldsymbol{\nabla})$; cellobiose $(\theta)$; maltose $(O)$; xylose $(\boldsymbol{\Delta})$; galactose $(\Delta)$; acide gluconique $(\bullet)$; ribose $(\bullet)$; lactose $(\nabla)$.

Figure 2. Relationship between specific growth rate and amount of pyruvate transformed to lactate during batch cultures of $L$. lactis NCDO 2118 on various sugars. Fructose ( ) man-

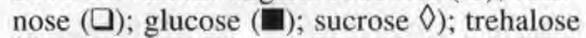
$(\boldsymbol{\nabla})$; cellobiose $(0)$; maltose $(O)$; xylose $(\boldsymbol{\Delta})$; galactose $(\Delta)$; gluconic acid $(\bullet)$; ribose $(\bullet)$; and lactose $(\nabla)$.

effet, sur lactose, la vitesse de consommation de substrat est très faible et le métabolisme est mixte sans production significative de lactate, tandis que sur glucose, la vitesse spécifique de consommation du sucre est élevée et le métabolisme est homolactique. Le métabolisme, ainsi que la vitesse de consommation du galactose, sont intermédiaires à ceux obtenus sur glucose et lactose. Une relation linéaire entre le taux de croissance et la vitesse de production d'ATP a déjà été démontrée chez L. lactis, dans le cas du métabolisme homolactique [8]. Lors du métabolisme mixte, sur galactose et lactose, le taux de croissance résulte de la vitesse d'assimilation du sucre qui détermine la quantité d'ATP produite par la glycolyse, mais 
aussi de la vitesse de production d'acétate qui provoque la synthèse d'ATP supplémentaire. Ainsi, en raison de la forte production d'acétate sur lactose, aucune différence significative de taux de croissance n'est mesurée entre les deux substrats malgré une plus faible vitesse de consommation du sucre (tableau I).

\subsection{Aspect enzymatique}

Pour mieux caractériser le shift homolactique-mixte, les concentrations d'intermédiaires glycolytiques ont été mesurées sur glucose, galactose et lactose, en phase exponentielle de croissance. Les concentrations de fructose-1-6-diphosphate et de trioses-phosphates (glycéraldehyde-3-phosphate et dihydroxyacétone-phosphate) sont élevées sur glucose mais considérablement plus faibles sur galactose ou lactose (tableau II). Sur ces deux derniers substrats, les faibles valeurs de pool tout le long de la glycolyse semblent en accord avec un flux de sucre limitant. En effet, étant d'origine végétale, la souche étudiée se caractérise par l'absence de plasmide lactose qui code pour le système de transport lactose phosphotransférase et les enzymes de la voie du tagatose (voie d'assimilation du galactose ou de la partie galactose du lactose). Ainsi, ne possédant que la voie de Leloir comme alternative, l'hypothèse d'un flux de carbone limitant semble légitime étant donné les faibles valeurs des vitesses de consommation de galactose et surtout de lactose. Sur glucose, par contre, le profil d'intermédiaires glycolytiques semble indiquer un blocage au niveau de la glycéraldehyde-3-phosphate déshydrogénase car tous les pools en amont de l'enzyme sont élevés et ceux en aval faibles. Un contrôle de la glycolyse par cette enzyme avait déjà été supposé sur des cellules non proliférantes de $L$. lactis [10]. Pour le vérifier d'un point de vue enzymatique, le dosage de la glycéraldehyde-3-phosphate déshydrogénase a été effectué sur chacun des trois substrats, mais un large excès d'enzyme a été trouvé par rapport au flux qu'elle assure réellement. Pour déterminer l'activité de l'enzyme in vivo, une étude de la régulation de l'activité de la glycéraldehyde-3phosphate déshydrogénase a été entreprise. Comme pour la glycéraldehyde-3-phosphate déshydrogénase de Clostridium acetobutylicum [5], un très strict contrôle négatif de l'enzyme par le ratio NADH/NAD

Tableau I. Vitesses spécifiques maximales de croissance ( $\mu$ ), de consommation du sucre (qs) et de production des produits de fermentation $(v)$ pendant une croissance discontinue de $L$. lactis NCDO 2118 sur glucose, galactose ou lactose.

Table I. Specific rates of growth $(\mu)$, sugar consumption (qs), and product formation ( $v$ ) during batch growth of $L$. lactis on glucose, galactose, or lactose.

\begin{tabular}{lccc}
\hline & Glucose & Galactose & Lactose \\
\hline$\mu\left(\mathrm{h}^{-1}\right)$ & 0,58 & 0,23 & 0,20 \\
$\mathrm{qs}\left(\mathrm{mmol} \mathrm{C6} \cdot \mathrm{g}^{-1} \cdot \mathrm{h}^{-1}\right)^{1}$ & 19 & 10,6 & 7,2 \\
${ }^{\mathrm{v}}$ lactate $\left(\mathrm{mmol} \cdot \mathrm{g}^{-1} \cdot \mathrm{h}^{-1}\right)$ & 32,0 & 11,3 & 0,5 \\
${ }^{v}$ formiate $\left(\mathrm{mmol} \cdot \mathrm{g}^{-1} \cdot \mathrm{h}^{-1}\right)$ & 2,0 & 6,1 & 11,3 \\
${ }^{v}$ acétate $\left(\mathrm{mmol} \cdot \mathrm{g}^{-1} \cdot \mathrm{h}^{-1}\right)$ & 0,7 & 2,8 & 5,0 \\
${ }^{v}$ éthanol $\left(\mathrm{mmol} \cdot \mathrm{g}^{-1} \cdot \mathrm{h}^{-1}\right)$ & 0,8 & 2,6 & 5,6 \\
\hline
\end{tabular}

${ }^{1}$ Vitesses spécifiques exprimées en équivalents hexoses.

' Sugar consumption rates are expressed as hexose equivalents. 
Tableau II. Concentrations en intermédiaires glycolytiques (mmol. $\left.\mathrm{L}^{-1}\right)$ lors de croissances discontinues de L. lactis NCDO 2118 sur glucose, galactose ou lactose. Les cellules sont prélevées en phase exponentielle et les valeurs indiquées dans le tableau correspondent à une moyenne de quatre mesures réalisées sur des extraits différents.

Table II. Glycolytic metabolite concentrations in cells harvested throughout the exponential growth phase of $L$. lactis on various sugars. Each value is an average of at least four samples taken during the exponential growth phase.

\begin{tabular}{lccc}
\hline & Glucose & Galactose & Lactose \\
\hline Glucose-6-phosphate & $25 \pm 3$ & $25 \pm 3$ & $15 \pm 2$ \\
Fructose-6-phosphate & $1,8 \pm 0,6$ & $5,9 \pm 0,5$ & $2,9 \pm 0,6$ \\
Fructose -1,6-diphosphate & $118 \pm 15$ & $36 \pm 16$ & $26 \pm 9$ \\
Dihydroxyacetone-phosphate & $21 \pm 3$ & $<0,6$ & $<0,6$ \\
Glycéraldehyde-3-phosphate & $6 \pm 2$ & $<0,6$ & $<0,6$ \\
1,3-diphosphoglycérate & $<0,6$ & $<0,6$ & $<0,6$ \\
3-phosphoglycérate & $<0,6$ & $<0,6$ & $<0,6$ \\
Phosphoénolpyruvate & $25 \pm 10$ & $<0,6$ & $<0,6$ \\
Phosphate inorganique & $\mathrm{ND}$ & $78 \pm 2$ & $\mathrm{ND}$ \\
\hline
\end{tabular}

ND : non déterminé.

ND : not determined.

a été mis en évidence (figure 3). Parallèlement, un dosage du ratio NADH/NAD in vivo a été réalisé sur les trois substrats, et une nette augmentation de ce ratio a été mesurée du lactose au galactose et au glucose, cette variation ayant pu être corrélée à la vitesse de consommation du sucre (figure 3). Ainsi, en prenant en compte 1'inhibition de l'enzyme par le ratio NADH/NAD, la quantité d'enzyme est environ cinq fois plus importante que le flux sur lactose et galactose tandis qu'elle suffit tout juste à assurer le flux sur glucose. Ce résultat confirme la limitation au niveau de la glycéraldehyde-3-phosphate déshydrogénase dans le cas du métabolisme du glucose, la forte accumulation des pools de fructose-1,6-diphosphate et de trioses- phosphates étant la conséquence directe de cette limitation.

Bien que le fructose-1,6-diphosphate, principal activateur de la lactate déshydrogénase $[13,15]$ ait été considéré jusqu'à présent comme le métabolite clé de la fermentation homolactique, son rôle dans le shift homolactique-mixte a pu être écarté. En effet, la concentration

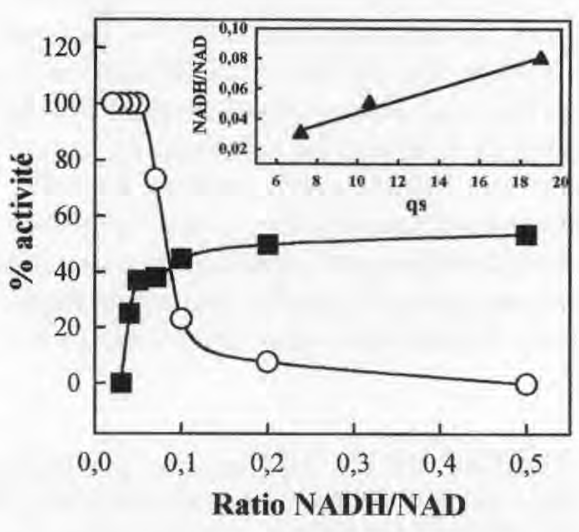

Figure 3. Effet du ratio NADH/NAD sur l'activité spécifique de la glycéraldehyde-3-phosphate déshydrogénase $(O)$ et la lactate déshydrogénase (四) de L. lactis NCDO 2118. L'encart montre comment le ratio NADH/NAD (A) est corrélé à la vitesse spécifique de consommation de substrat (qs) exprimée en mmol.g $g^{-1} \cdot h^{-1}$.

Figure 3. Effect of the NADH/NAD ratio on activities of glyceraldehyde-3-phosphate dehydrogenase $(O)$ and lactate dehydrogenase (of L. lactis NCDO 2118. The insert shows the relationship between the NADH/NAD ratio $(\boldsymbol{\Delta})$ and the specific rate of sugar consumption $\left(\mathrm{mmol} \cdot \mathrm{g}^{-1} \cdot \mathrm{h}^{-1}\right)$. 
de ce métabolite, très élevée sur glucose (118 mmol. $\left.\mathrm{L}^{-1}\right)$, diminue sur galactose ou lactose $\left(27 \mathrm{mmol}^{-1} \mathrm{~L}^{-1}\right)$, mais pas suffisamment pour provoquer l'inactivation de la lactate déshydrogénase. La constante d'activation de la lactate déshydrogénase chez $L$. lactis est en effet extrêmement faible, d'environ $2 \mu \mathrm{mol} . \mathrm{L}^{-1}$ (figure 4), et dans ces conditions, les concentrations intracellulaires de fructose-1,6-diphosphate suffisent sur les trois substrats à activer pleinement l'enzyme. Simultanément, la concentration d'enzyme diminue d'un facteur deux alors que le flux à travers la

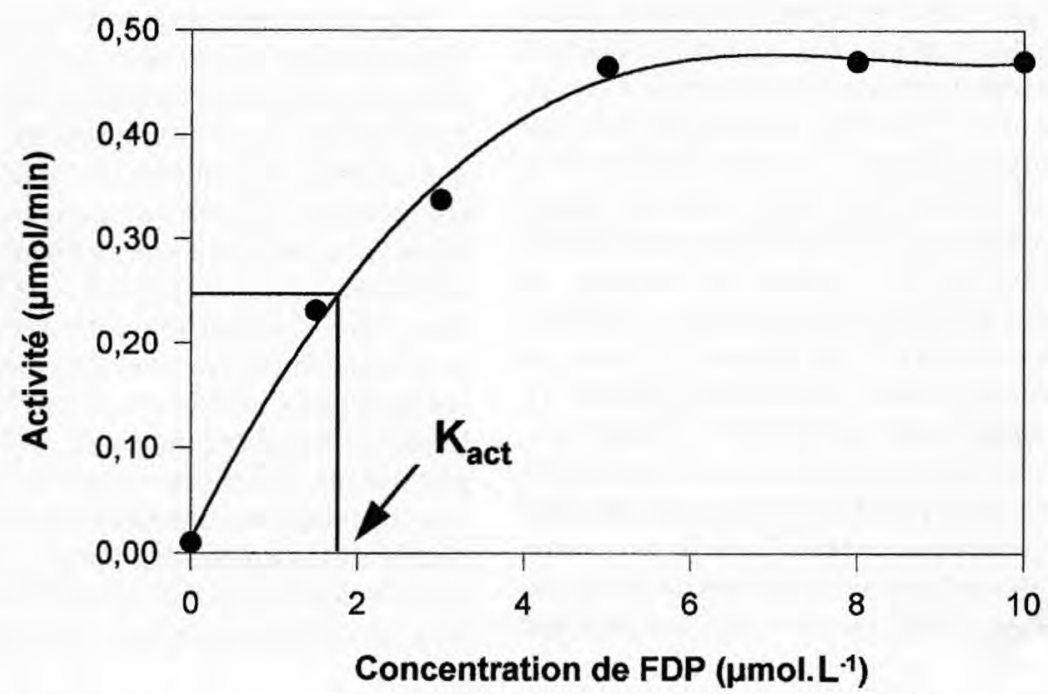

Figure 4. Courbe d'activation de la lactate déshydrogénase de L. lactis NCDO 2118 par le fructose-1,6-diphosphate.

Figure 4. Fructose-1,6-diphosphate activation curve for lactate dehydrogenase activity of $L$. lactis NCDO 2118.

Tableau III. Effet du phosphate inorganique sur la constante d'activation de la lactate déshydrogénase de L. lactis NCDO 2118 par le fructose-1,6-diphosphate.

Table III. Effect of the inorganic phosphate concentration on the fructose-1,6-diphosphate activation constant for lactate dehydrogenase of L. lactis NCDO 2118.

\begin{tabular}{lllrl}
\hline Phosphate inorganique $\left(\mathrm{mmol} . \mathrm{L}^{-1}\right)$ & 0 & 5 & 100 & 500 \\
Constante d'activation $\left(\mu \mathrm{mol} . \mathrm{L}^{-1}\right)$ & 2 & 2,5 & 40 & 130 \\
\hline
\end{tabular}

Tableau IV. Effet inhibiteur du phosphoénolpyruvate sur la lactate déshydrogénase de L. lactis NCDO 2118.

Table IV. Inhibitory effect of phosphoenolpyruvate on L. lactis NCDO 2118 lactate dehydrogenase activity.

\begin{tabular}{lrrrrr}
\hline Phosphoénolpyruvate (mmol. $\mathrm{L}^{-1}$ ) & 0 & 5 & 10 & 20 & 40 \\
Activité lactate déshydrogénase $(\%)$ & 100 & 100 & 100 & 80 & 70 \\
\hline
\end{tabular}


lactate déshydrogénase baisse de $98 \%$ du glucose au lactose. La quantité d'enzyme ne peut donc pas expliquer à elle seule ce shift métabolique, ce qui prouve qu'un contrôle de l'activité de la lactate déshydrogénase, autre que par le fructose-1,6diphosphate, doit avoir lieu in vivo. Aucun effet régulateur classiquement attendu [14], tel que l'inhibition par le phosphate inorganique (tableau III) ou le phosphoénolpyruvate (tableau IV), ne semble avoir de rôle in vivo. En effet, l'activité de la lactate déshydrogénase n'est pas significativement altérée pour des concentrations d'inhibiteurs bien supérieures aux concentrations physiologiques. En revanche, la lactate déshydrogénase de $L$. lactis NCDO 2118 est régulée de façon très stricte in vitro par le ratio NADH/NAD (figure 3), et compte tenu des ratio mesurés sur chacun des trois substrats, il a été démontré que cette régulation participait au shift homolactique-mixte. En effet, en corrigeant l'activité de la lactate déshydrogénase par l'effet du ratio sur l'enzyme, une corrélation parfaite a été obtenue entre cette activité et le flux réel passant par l'enzyme. La concentration intracellulaire de pyruvate, le substrat de la lactate déshydrogénase, a pu être modélisée pour satisfaire le flux: Une valeur non saturante estimée à $1 \mathrm{mmol} . \mathrm{L}^{-1}$ par rapport à un $\mathrm{Km}$ de $3 \mathrm{mmol} . \mathrm{L}^{-1}$ a ainsi été obtenue [4].

Pour orienter le flux, soit vers la pyruvate formiate lyase, soit vers la lactate déshydrogénase, le contrôle négatif d'une enzyme doit avoir lieu en même temps que la levée du contrôle de l'enzyme en compétition. Concernant la pyruvate formiate lyase, en plus d'une augmentation de la concentration d'enzyme d'un facteur deux, un baisse sensible de la concentration intracellulaire des trioses-phosphates, ses principaux inhibiteurs, se produit dans le cas du métabolisme mixte. Ainsi, sur glucose une inhibition stricte de la pyruvate formiate lyase par les trioses-phosphates a été démontrée, compte tenu des fortes valeurs de pools (soit $21 \mathrm{mmol} . \mathrm{L}^{-1}$ pour le dihydroxyacétone phosphate et

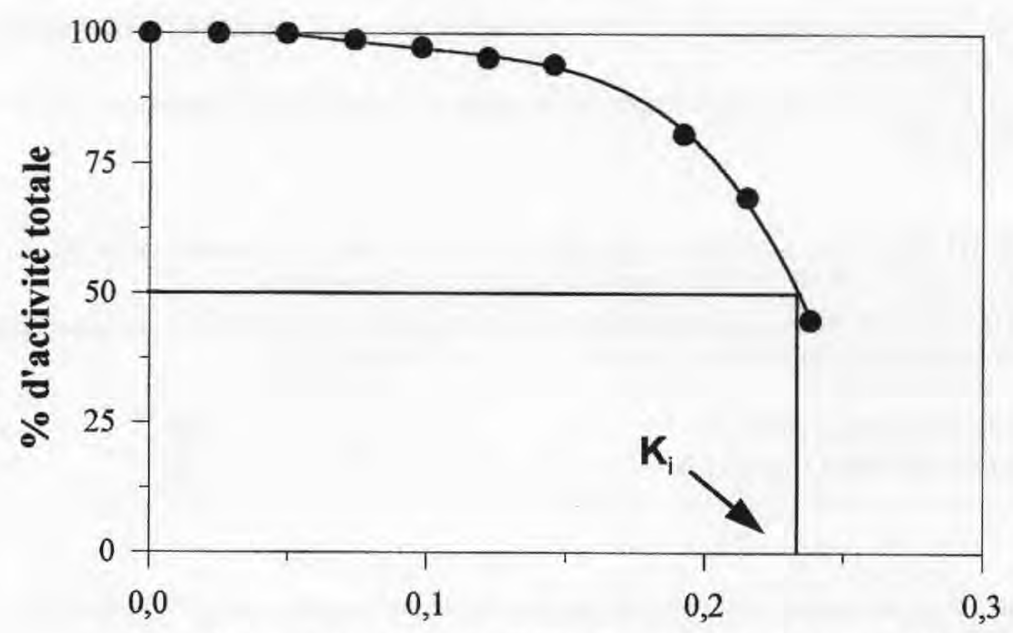

Concentration de DHAP ou GAP (mmol.L-1)

Figure 5. Courbe d'inhibition de la pyruvate formiate lyase de L. lactis NCDO 2118 par le glycéraldéhyde-3-phosphate ou le dihydroxyacétone phosphate.

Figure 5. Inhibition of pyruvate formate lyase activity from $L$. lactis NCDO 2118 by glyceraldehyde3-phosphate and dihydroxyacetone phosphate. 
$6 \mathrm{mmol} . \mathrm{L}^{-1}$ pour le glycéraldehyde-3phosphate) par rapport à la constante d'inhibition de l'enzyme $\left(0,2-0,3\right.$ mmol. $\mathrm{L}^{-1}$ pour chacun des deux trioses-phosphates, figure 5).

Une plus faible valeur de $\mathrm{Km}$ pour la pyruvate formiate lyase, comparée à la lactate déshydrogénase, montre aussi le rôle de la concentration intracellulaire de pyruvate dans le shift homolactique-mixte, car à faible concentration de pyruvate, la pyruvate formiate lyase pourra être plus compétitive malgré une vitesse maximale apparemment plus faible [4].

\subsection{Modèle de régulation proposé}

Cette étude a permis de minimiser chez L. lactis le rôle du fructose-1,6-diphosphate dans le shift homolactique-mixte, malgré l'importance qu'on lui attribue généralement. Un modèle plus cohérent basé sur la régulation du métabolisme central par le ratio NADH/NAD a été proposé. Dans le cas d'un flux glycolytique élevé, un fort ratio NADH/NAD provoque une limitation au niveau de la glycéraldehyde-3-phosphate déshydrogénase qui engendre des pools de fructose-1,6-diphosphate et de trioses-phosphates élevés. Dans ces conditions, la lactate déshydrogénase est activée par le fort ratio NADH/NAD, tandis que la pyruvate formiate lyase, faiblement induite, est inhibée par les triosesphosphates. Le métabolisme est alors homolactique. Inversement, lorsque le flux glycolytique diminue, l'activité de la lactate déshydrogénase est faible en raison d'une plus faible concentration d'enzyme et surtout du faible ratio NADH/NAD. De plus, le contrôle négatif de la glycéraldehyde-3-phosphate déshydrogénase par le ratio NADH/NAD est levé, évitant l'accumulation importante de trioses-phosphates inhibiteurs de la pyruvate formiate lyase. Le métabolisme s'oriente alors vers un métabolisme mixte.

\subsection{Validation du modèle}

Ce modèle original de régulation a été établi par la comparaison du comportement métabolique de la souche étudiée sur divers substrats, mais dans un milieu de culture identique. Une approche complémentaire a consisté à étudier la croissance et le métabolisme de cette souche sur des milieux synthétiques de composition différente. Le galactose a été choisi comme substrat pour cette étude car il offre un métabolisme mixte où lactate et produits dérivés de la pyruvate formiate lyase sont produits en quantité significative. Il s'agit donc ici de faire varier le flux anabolique et d'étudier les conséquences sur le flux catabolique et la distribution des produits formés.

Comme attendu d'après le modèle de régulation, dans le milieu le plus simplifié, le flux anabolique est limitant, et le métabolisme qui en résulte est homolactique. En effet, le flux catabolique, même s'il est limité en raison de l'absence du plasmide lactose, reste excédentaire par rapport au flux anabolique. Au contraire, sur un milieu plus riche permettant une bonne croissance, l'anabolisme n'est plus limitant. Dans ce cas, l'étape la plus lente est la vitesse d'assimilation du galactose, et le flux de carbone est alors orienté vers un métabolisme mixte. Ces données montrent clairement la relation intime qui unit l'ensemble des voies métaboliques mises en cuvre lors de la croissance du microorganisme, où flux catabolique et anabolique s'adaptent l'un à l'autre pour un fonctionnement optimal de l'ensemble du réseau métabolique en fonction des contraintes imposées par l'environnement.

Afin de généraliser le modèle proposé, l'étude de la régulation du métabolisme d'une autre bactérie lactique a été entreprise avec une stratégie identique à celle menée précédemment. Pour cela, la caractérisation macro et micro-cinétique de deux souches de $L$. lactis subsp cremo- 
ris, MG 1820 et MG 1363, différentes par la présence ou non du plasmide lactose, a été effectuée. Cette étude a permis de montrer que, comme pour la souche d'origine végétale, trois paramètres orientent le flux de pyruvate entre les divers produits de fermentation : la concentration d'enzyme, les caractéristiques cinétiques de la lactate déshydrogénase et de la pyruvate formiate lyase, et surtout la régulation de ces deux activités par le ratio NADH/NAD et les trioses-phosphates respectivement. Le modèle de régulation proposé pour $L$. lactis NCDO 2118 est donc généralisable à d'autres souches de L. lactis, y compris de la sous-espèce cremoris.

$\mathrm{Vu}$ l'importance du rapport NADH/NAD, il devient très intéressant d'étudier maintenant les perturbations métaboliques qui interviennent en condition aérobie. En effet, la possibilité de moduler le métabolisme du pyruvate dans les souches possédant de fortes activités NADH oxydase présente un intérêt potentiel pour la production d'arôme issu du pyruvate tel que le diacétyle.

\section{INFLUENCE DES COSUBSTRATS}

Le métabolisme des bactéries lactiques peut être modulé par un apport nutritionnel autre que le sucre, notamment les cosubstrats. C'est le cas, en particulier, de l'apport de citrate qui modifie la répartition du flux de pyruvate chez L. lactis. Le citrate génère, en effet, du pyruvate sans production de coenzymes réduits et, dans certaines conditions de fermentation, ce flux est orienté vers la production de diacétyle, acétoine et butanediol par l'intermédiaire de l'acétolactate $[6,11]$. Dans certains cas cependant, l'apport de cosubstrat ne provoque pas une réorientation du métabolisme vers d'autres voies métaboliques, mais le flux de carbone supplémentaire se répartit entre les voies déjà existantes, au même titre que le flux cata- bolique. Une telle situation est rencontrée chez L. lactis cultivée sur un milieu minimum contenant peu d'acides aminés, dans lequel une consommation de sérine largement supérieure aux besoins anaboliques est observée. Cette sérine est massivement désaminée en pyruvate qui se répartit majoritairement vers la production de lactate, comme le flux issu du glucose [8]. La sérine joue alors le rôle d'un cosubstrat, qui crée un apport de carbone supplémentaire au niveau du pyruvate, mais aussi d'un accepteur d'électrons supplémentaire avec pour conséquence une redistribution des produits issus de l'acétylCoA (acétate et éthanol). Une absence de production d'éthanol en faveur de la synthèse d'acétate est en effet observée en raison de la synthèse de lactate supplémentaire provoquée par l'assimilation de la sérine.

\section{CONCLUSION}

Le métabolisme des bactéries lactiques est très sensible aux variations de l'environnement. Malgré l'importance que revêt une bonne compréhension de ces phénomènes pour l'exploitation optimale de ces bactéries, peu d'études ont été réalisées dans ce domaine. Face à ces contraintes environnementales, le comportement des lactocoques commence tout juste à être décrit et n'est généralement pas bien expliqué. Les techniques disponibles de génétique et de biochimie devraient permettre de mieux cerner les phénomènes observés en physiologie et d'améliorer rapidement les connaissances sur ce sujet. A terme, ces résultats devraient donner lieu à une meilleure mise en œuvre des bactéries lactiques, ainsi qu'à de nouvelles stratégies de sélection ou de construction de souches possédant les caractéristiques souhaitées pour l'industrie laitière, aussi bien au niveau des profils de métabolites, que dans leur comportement en conditions de cultures défavorables. 


\section{RÉFÉRENCES}

[1] Baronofsky J.J., Schreurs W.J.A., Kashket E.R., Uncoupling by acetic acid limits growth of and acetogenesis by Clostridium thermoaceticum, Appl. Environ. Microbiol. 48 (1984) 1134-1139.

[2] Cocaign-Bousquet M., Garrigues C., Novak L., Lindley N.D., Loubière P., Rational development of a simple synthetic medium for the sustained growth of Lactococcus lactis, J. Appl. Bacteriol. 79 (1995) 108-116.

[3] Cocaign-Bousquet M., Garrigues C., Loubière P., Lindley N.D., Physiology of pyruvate metabolism in Lactococcus lactis, Antonie van Leeuwenhoek 70 (1996) 253-267.

[4] Garrigues C., Loubière P., Lindley N.D., Cocaign-Bousquet M., Control of the shift from homolactic to mixed acid fermentation in Lactococcus lactis: predominant role of the NADH/NAD ratio, J. Bacteriol. 179 (1997) 5282-5287.

[5] Girbal L., Soucaille P., Regulation of Clostridium acetobutylicum metabolism revealed by mixed substrate chemostat cultures: role of NADH/NAD ratio and ATP pool, J. Bacteriol. 176 (1994) 6433-6438.

[6] Hugenholtz J., Starrenburg M.J.C., Diacetyl production by different strains of Lactococcus lactis subsp. lactis var. diacetylactis and Leuconostoc spp., Appl. Microbiol. Biotechnol. 38 (1992) 17-22.

[7] Loubière P., Cocaign-Bousquet M., Matos J., Goma G., Lindley N.D., Influence of endproduct inhibition and nutrient limitations on the growth of Lactococcus lactis subsp. lactis, J. Appl. Bacteriol. 82 (1997) 95-100.
[8] Novak L., Cocaign-Bousquet M., Lindley N.D., Loubière P., Metabolism and energetics of Lactococcus lactis during growth in complex or synthetic media, Appl. Environ. Microbiol. 63 (1997) 2665-2670.

[9] Novak L., Cocaign-Bousquet M., Lindley N.D., Loubière P., Cométabolisme sucre-acides aminés chez Lactococcus lactis. Lait 78 (1998).

[10] Poolman B., Bosman B., Kiers J., Konings W.N., Control of glycolysis by glyceraldehyde-3phosphate dehydrogenase in Streptococcus cremoris and Streptococcus lactis, J. Bacteriol. 169 (1987) 5887-5890.

[11] Ramos A., Jordan K.N., Cogan T.M., Santos H., 13C Nuclear magnetic resonance studies of citrate and glucose cometabolism by Lactococcus lactis, Appl. Environ. Microbiol. 60 (1994) 1739-1748.

[12] Thomas T.D., Regulation of lactose fermentation in group N. streptococci, Appl. Environ. Microbiol. 32 (1976) 474478.

[13] Thomas T.D., Ellwood D.C., Longyear M.C., Change from homo- to heterolactic fermentation by Streptococcus lactis resulting from glucose limitation in anaerobic chemostat cultures, J. Bacteriol. 138 (1979) 109-117.

[14] Yamada T., Regulation of glycolysis in streptococci. In: Sugar transport and metabolism in gram-positive bacteria. (J. Reizer, A. Peterkofsky eds) Ellis Horwood Series in Biochemistry and Biotechnology. Chichester, England, 1987, 69-93.

[15] Yamada T., Carlsson J., Regulation of lactate dehydrogenase and change of fermentation products in streptococci, J. Bacteriol. 124 (1975) 55-61. 